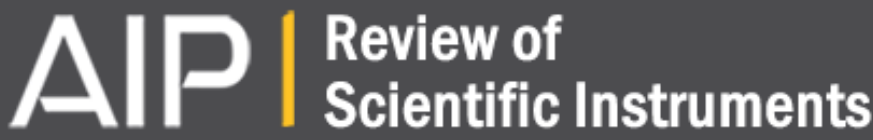

Time-resolved pump and probe x-ray absorption fine structure spectroscopy at beamline P11 at PETRA III

D. Göries, B. Dicke, P. Roedig, N. Stübe, J. Meyer, A. Galler, W. Gawelda, A. Britz, P. Geßler, H. Sotoudi Namin, A. Beckmann, M. Schlie, M. Warmer, M. Naumova, C. Bressler, M. Rübhausen, E. Weckert, and A. Meents

Citation: Review of Scientific Instruments 87, 053116 (2016); doi: 10.1063/1.4948596

View online: http://dx.doi.org/10.1063/1.4948596

View Table of Contents: http://scitation.aip.org/content/aip/journal/rsi/87/5?ver=pdfcov

Published by the AIP Publishing

\section{Articles you may be interested in}

Time-resolved near-edge $x$-ray absorption fine structure spectroscopy on photo-induced phase transitions using a tabletop soft-x-ray spectrometer

Rev. Sci. Instrum. 83, 053110 (2012); 10.1063/1.4718936

Hydrogen bonding in acetone clusters probed by near-edge x-ray absorption fine structure spectroscopy in the carbon and oxygen $\mathrm{K}$-edge regions

J. Chem. Phys. 131, 174311 (2009); 10.1063/1.3257962

Solving the structure of reaction intermediates by time-resolved synchrotron x-ray absorption spectroscopy J. Chem. Phys. 129, 234502 (2008); 10.1063/1.3040271

Towards structural dynamics in condensed chemical systems exploiting ultrafast time-resolved x-ray absorption spectroscopy

J. Chem. Phys. 116, 2955 (2002); 10.1063/1.1435618

Differentiation of biological hydroxyapatite compounds by infrared spectroscopy, x-ray diffraction and extended $\mathrm{x}$-ray absorption fine structure

J. Appl. Phys. 90, 6440 (2001); 10.1063/1.1408267

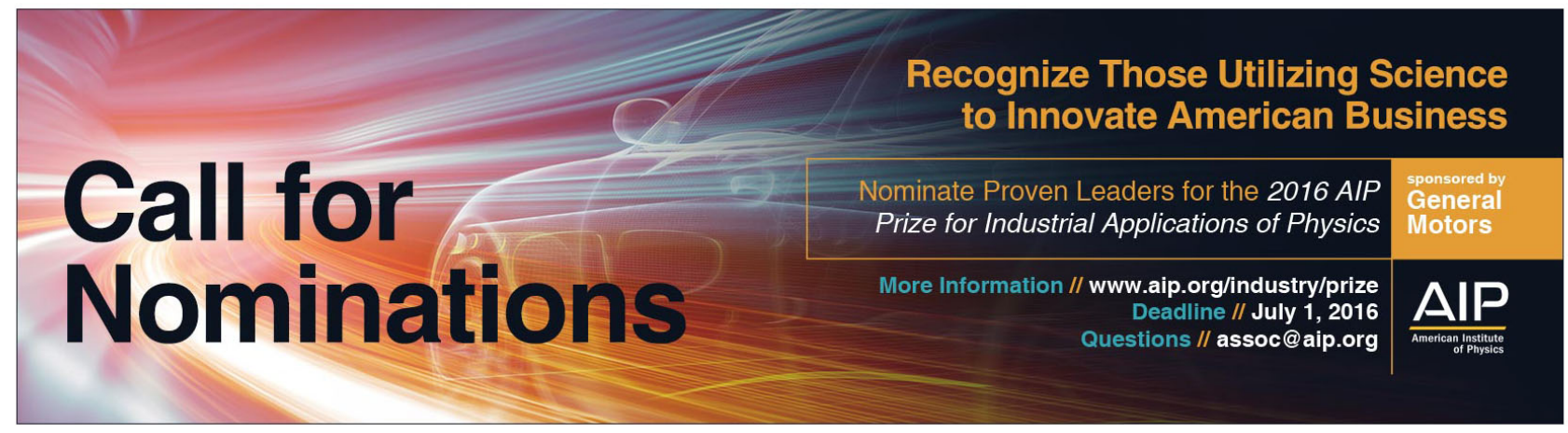




\title{
Time-resolved pump and probe x-ray absorption fine structure spectroscopy at beamline P11 at PETRA III
}

\author{
D. Göries, ${ }^{1, a)}$ B. Dicke,${ }^{2}$ P. Roedig, ${ }^{1}$ N. Stübe,${ }^{1}$ J. Meyer,${ }^{1}$ A. Galler,${ }^{3}$ W. Gawelda,${ }^{3}$ \\ A. Britz,,$^{3,4}$ P. Geßler, ${ }^{3}$ H. Sotoudi Namin, ${ }^{3}$ A. Beckmann, ${ }^{3}$ M. Schlie, ${ }^{5}$ M. Warmer, ${ }^{1}$ \\ M. Naumova, ${ }^{2}$ C. Bressler, ${ }^{3,4}$ M. Rübhausen, ${ }^{2}$ E. Weckert, ${ }^{1}$ and A. Meents ${ }^{1, b)}$ \\ ${ }^{1}$ DESY Photon Science, Deutsches Elektronen-Synchrotron (DESY), Notkestraße 85, 22607 Hamburg, Germany \\ ${ }^{2}$ Center for Free-Electron Laser Science (CFEL), Luruper Chaussee 149, 22761 Hamburg, Germany \\ ${ }^{3}$ European XFEL, Albert-Einstein Ring 19, 22761 Hamburg, Germany \\ ${ }^{4}$ The Hamburg Centre for Ultrafast Imaging, Luruper Chaussee 149, 22761 Hamburg, Germany \\ ${ }^{5}$ Institut für Experimentalphysik, University of Hamburg, Luruper Chaussee 149, 22761 Hamburg, Germany
}

(Received 29 October 2015; accepted 21 April 2016; published online 17 May 2016)

\begin{abstract}
We report about the development and implementation of a new setup for time-resolved X-ray absorption fine structure spectroscopy at beamline P11 utilizing the outstanding source properties of the low-emittance PETRA III synchrotron storage ring in Hamburg. Using a high intensity micrometer-sized X-ray beam in combination with two positional feedback systems, measurements were performed on the transition metal complex fac-Tris[2-phenylpyridinato-C2,N]iridium(III) also referred to as fac-Ir(ppy) 3 . This compound is a representative of the phosphorescent iridium(III) complexes, which play an important role in organic light emitting diode (OLED) technology. The experiment could directly prove the anticipated photoinduced charge transfer reaction. Our results further reveal that the temporal resolution of the experiment is limited by the PETRA III X-ray bunch length of $\sim 103$ ps full width at half maximum (FWHM). Published by AIP Publishing. [http://dx.doi.org/10.1063/1.4948596]
\end{abstract}

\section{INTRODUCTION}

At low-emittance synchrotron sources, it has become possible to extent well-established X-ray techniques such as $\mathrm{X}$-ray absorption fine structure spectroscopy (XAFS), X-ray emission spectroscopy, and X-ray crystallography to the time domain and to perform such experiments in an acceptable time requiring small sample amounts only. Such time-resolved experiments are typically performed using a laser pump and Xray probe configuration to obtain information about transient structures. ${ }^{1-4}$ Such information about the dynamics of the electronic and structural properties of materials is essential to understand their function. In a pump-probe experiment, a laser pulse excites the system of interest, while a following $\mathrm{X}$-ray pulse-at a certain time delay with respect to the laser pump pulse-probes the sample and reads out the photoinduced changes. The excited state dynamics can be followed by adjusting the time delay between the laser and X-ray pulse, which allows to investigate the temporal evolution of the structural changes and to identify intermediate excited states. ${ }^{1,2}$ Time-resolved X-ray absorption fine structure spectroscopy (XAFS) has been well established at third generation synchrotron sources in the picosecond time domain and has been demonstrated to be a powerful tool. ${ }^{5-10}$

This method provides access to photoinduced bond length changes ${ }^{11-14}$ and is further an ideally suited technique to investigate charge transfer reactions in transition metal complexes. ${ }^{15,16}$

\footnotetext{
a)Electronic mail: dennis.goeries@desy.de.

b)Electronic mail: alke.meents@ desy.de
}

X-ray absorption spectra are primarily characterized by element-specific absorption edges. The spectra can be divided into two energy regions of interest, namely, the X-ray absorption near-edge structure (XANES) and the extended Xray absorption fine structure (EXAFS). The XANES region primarily provides electronic information of the element of interest, e.g., the oxidation state, but can be also used to obtain some structural information. ${ }^{17-19}$ The EXAFS region contains further detailed structural information such as the geometry and the nearest neighbor distances of the absorber. ${ }^{20-22}$

We have developed and established a new setup for time-resolved X-ray absorption fine structure spectroscopy experiments at beamline P11 at the PETRA III storage ring at DESY in Hamburg. ${ }^{23,24}$ Due to the low emittance of PETRA III and the focusing capabilities of beamline P11, this setup is able to work with micrometer beam sizes, which allow minimizing the required sample volume. The required sample amount is an important factor in many XAFS experiments, especially if the sample molecules suffer from radiation damage (either X-ray or laser induced). Special care was taken to guarantee longterm stable spatial overlap between sample jet, laser pump, and X-ray probe beam.

In this paper, we introduce the reader to the beamline P11 at PETRA III followed by a detailed explanation of our experimental XAFS setup. More setups for time-resolved XAFS have been installed at other synchrotrons sources, which are described in Refs. 8, 9, and 25-28.

In the second part, experimental results obtained with the new P11 time-resolved XAFS setup are presented. In a laserpump and X-ray-probe experiment, we were able to directly 
prove the anticipated photoinduced metal-to-ligand charge transfer (MLCT) reaction in the $\operatorname{Ir}(\mathrm{ppy})_{3}$ metal complex, which is an important compound for flexible organic light emitting diode (OLED) technology. ${ }^{29,30}$

\section{BEAMLINE P11 AT PETRA III}

The PETRA III storage ring ${ }^{31}$ in Hamburg provides a horizontal emittance of $1 \mathrm{~nm}$ rad and a vertical emittance of $10 \mathrm{pm} \mathrm{rad} \mathrm{(1 \%} \mathrm{coupling)} \mathrm{and} \mathrm{has} \mathrm{a} \mathrm{circumference} \mathrm{of}$ $2304 \mathrm{~m}$. The operating master frequency is $\sim 500 \mathrm{MHz}$ and the synchrotron provides 3840 buckets with a revolution frequency of $\sim 130 \mathrm{kHz}$ and a bucket separation of $2 \mathrm{~ns}$. The synchrotron can be operated at different filling patterns of 40, 60, 240, and 960 electron bunches with a nominal pulse duration of $\sim 103$ ps full width at half maximum (FWHM) for the experiments. ${ }^{31}$ PETRA III is typically operated in top-up mode with a current of $100 \mathrm{~mA}$, which is equally distributed among all bunches for the respective filling pattern.

Time-resolved XAFS measurements are ideally performed in 40 bunch mode since the repetition rate of the laser system inherently limits the maximal pump and data acquisition frequency. The corresponding bunch spacing of $192 \mathrm{~ns}$ is relatively large and facilitates the operation of gating electronics.

Beamline P11 at PETRA III is mainly dedicated to imaging and diffraction experiments of biological samples. The very flexible focusing optics and the large experimental hutch allow the hosting of many experiments such as the timeresolved XAFS setup described here. Beamline P11 covers a broad energy range from 2.4 up to $32 \mathrm{keV}$ and provides a high monochromatic photon flux $\left(\sim 1.65 \times 10^{13} \mathrm{ph} / \mathrm{s}\right.$ at $12 \mathrm{keV}$ (Si-111)), which corresponds to $\sim 3 \times 10^{6}$ photons per pulse in 40 bunch mode. First optical element is a liquid nitrogen cooled double crystal monochromator (DCM) for wavelength selection with either a pair of Si-111 or Si-311 crystals. The DCM is followed by a KB-mirror system consisting of two horizontally and one vertically deflecting X-ray mirrors at a distance of about $38 \mathrm{~m}$ from the source. All three mirrors are dynamically bendable, which allow tailoring the X-ray beam size at the position of the experiment according to the specific needs. In standard operation, these first three mirrors are used to generate a secondary source at a distance of $65.5 \mathrm{~m}$ from the source. A second KB-system also equipped with mechanical benders is located shortly before the sample position in the experimental hutch and is utilized to further demagnify the secondary source. This setup allows focusing the full photon flux from the source into a $4 \times 9 \mu \mathrm{m}^{2} \mathrm{X}$-ray spot $(\mathrm{v} \times \mathrm{h})$ (FWHM). Smaller beam sizes down to $1 \times 1 \mu \mathrm{m}^{2}$ with a photon flux of $2 \times 10^{11}$ can be realized by slitting down the secondary source.

Key element of the experimental hutch is an $8 \mathrm{~m}$ long granite block with a weight of 10 tons, which carries the different experiments. To reduce thermal drifts to a minimum, all significant heat sources such as power supplies and motor controllers are located outside the experimental hutch. The thermal stability of the experimental hutch measured over 25 hours is better than $\pm 0.1 \mathrm{~K}$. The humidity in the hutch is controlled to provide stable conditions for laser operation.

\section{INSTRUMENTS AND XAFS SETUP}

The XAFS setup at beamline P11 is installed directly behind the refocusing KB-system at a distance of $72.3 \mathrm{~m}$ from the source. An overview of the setup including the UV/VIS and $\mathrm{X}$-ray beam path is shown in Figure 1. The X-ray beam first passes through a silicon photodiode for $I_{0}$ measurements and further through a small hole in the on-axis microscope (OAM) before interacting with the sample. The sample is delivered by a liquid jet system, which is mounted on a three-axle translational motor stage. For X-ray fluorescence detection, two avalanche photodiodes (APDs) are mounted on a threeaxle positioning system at an angle of ninety degrees with respect to the incident beam. The UV/VIS pump laser pulses are guided to the sample by a set of mirrors (M) and focused by a lens shortly before the sample.

In the following, the major components of the setup are described. For more details, the reader is referred to Refs. 23, 24 , and 32 .

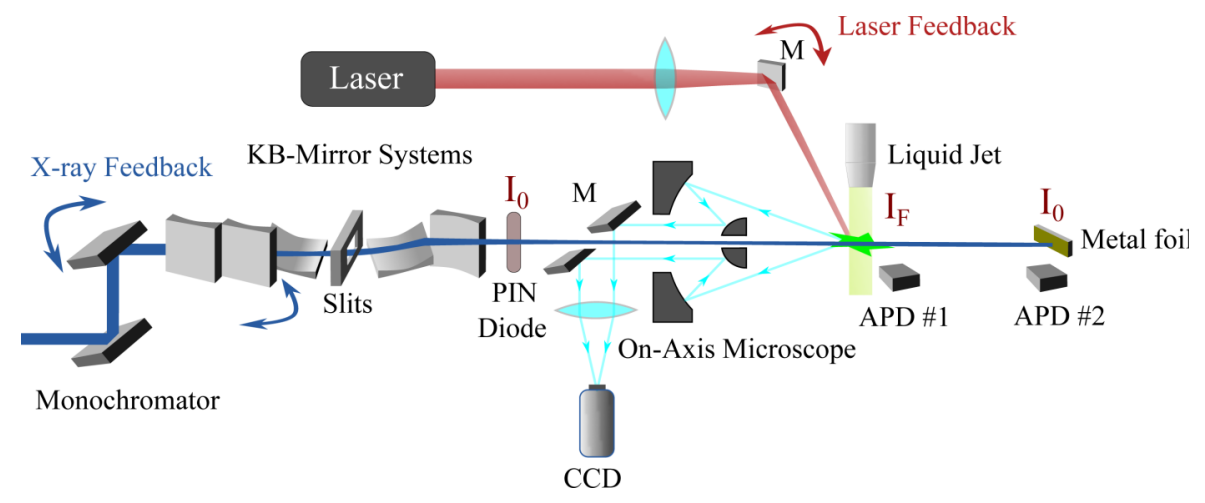

FIG. 1. Overview of the setup for time-resolved X-ray absorption fine structure spectroscopy (XAFS) experiments at beamline P11. The setup consists of an on-axis microscope with a drill hole for the X-rays to pass through, one motorized tower carrying the avalanche photo diode (APD) for X-ray fluorescence detection, and one motorized tower carrying the liquid jet equipped with YAG scintillator screen for beam visualization and a silicon edge for beam size characterization. More detailed descriptions of the individual components are provided in the main text. M: mirror; CCD: camera; $\mathrm{I}_{0}$ : incident intensity; $\mathrm{I}_{\mathrm{F}}$ : fluorescence signal; $\mathrm{I}_{1}$ : reference intensity, single shot calibration. 


\section{A. Ultra-short pulse laser system}

A commercial laser system from Light Conversion, Ltd. equipped with custom synchronization electronics from Menlo Systems is utilized to provide ultra-short UV/VIS pulses for photoexcitation of the sample. The PHAROS laser is an ytterbium based system featuring typical output pulses at a wavelength of $1030 \mathrm{~nm}$ and pulse durations of nominally $\sim 180$ fs. Depending on the sample conditions, e.g., preferred wavelength for photoexcitation, different secondary passive modules are utilized. The harmonic generator $H I R O$ provides the second $(515 \mathrm{~nm})$, third $(343 \mathrm{~nm})$, and fourth harmonic $(257 \mathrm{~nm})$ of the fundamental wavelength and has been designed to operate between $65 \mathrm{kHz}$ and $260 \mathrm{kHz}$ in order to allow for a fast data acquisition. Typical output pulse energies at a repetition rate of $65 \mathrm{kHz}$ are up to $92 \mu \mathrm{J}, 50 \mu \mathrm{J}, 25 \mu \mathrm{J}$, and $9 \mu \mathrm{J}$ for the fundamental, second, third, and fourth harmonic, respectively. These pulse energies are sufficient to excite a significant fraction of sample molecules.

The optical parametric amplifier ORPHEUS with the dedicated harmonic generator $L Y R A$ can be utilized to freely select any wavelength between $260 \mathrm{~nm}$ and $2600 \mathrm{~nm}$ at repetition rates between $5 \mathrm{kHz}$ and $10 \mathrm{kHz}$ providing pulse energies from $\sim 9 \mu \mathrm{J}$ in the ultraviolet to $\sim 100 \mu \mathrm{J}$ in the infrared photon energy regime. These smaller repetition rates are ideally suited for experiments with solid samples, which suffer from laser induced heating and cannot be exchanged in a very fast manner. Wavelengths tuning further allows adjusting the penetration depths of laser light into the sample according to the volume probed by the X-rays.

Synchronization to the synchrotron is achieved by a phase-lock of the laser oscillator to the PETRA III master reference of $500 \mathrm{MHz}$. In order to achieve a synchronized system, the oscillator $\sim 83.3 \mathrm{MHz}$ exhibits two piezoelectric actuators, which rapidly adjust the cavity length to balance the phase error between oscillator and synchrotron. The performance of the synchronization unit has been characterized to be long-term stable with a timing jitter of $\sim 1 \mathrm{ps}$ rms and the oscillator pulse train can be remotely phase-shifted in finesteps of $\sim 1.4$ ps. $^{23}$

In order to achieve temporal overlap between X-ray and laser pulses, the Pockels cell controlling the regenerative amplifier operation is triggered by multiple dividers of the PETRA III master reference. This allows selecting different pulses from the oscillator train to undergo the amplification process and enables a coarse time-shifting of $12 \mathrm{~ns}$.

The laser system is placed on a mobile optical table with a footprint of $0.8 \times 1.2 \mathrm{~m}^{2}$, which allows independent usage of the laser for other experiments at different beamlines or laser laboratories. For time-resolved experiments, the laser platform is moved into the experimental hutch and placed close to the granite block carrying the experiments.

\section{B. Micro-jet system}

The sample delivery system consists of a sample reservoir, a micro-fluidic pump, a glass nozzle, and a sample catcher. For the experiment, the sample is pumped from the reservoir by a pump and pressed through a glass nozzle with a diameter between $15 \mu \mathrm{m}$ and $300 \mu \mathrm{m}$ according to the needs of the exper-

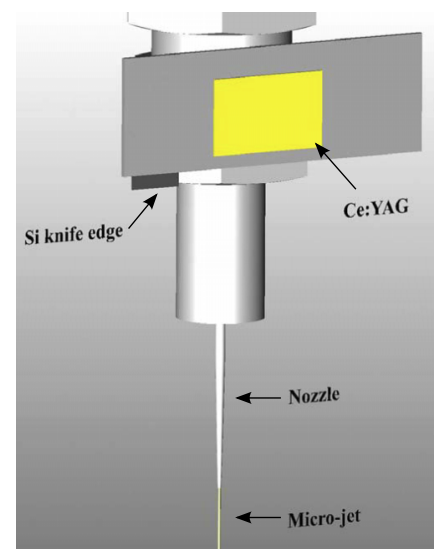

FIG. 2. Schematic drawing of the liquid jet setup equipped with its two different tools for X-ray and laser beam characterization. A cerium doped YAG scintillator crystal mounted above the jet nozzle allows visualizing both laser pump and X-ray probe beam. In addition, a sharp silicon edge is attached which can be moved into the beam to perform knife-edge scans for precise beam shape characterization.

iment. Using this approach, a stable laminar jet with velocities up to $10 \mathrm{~m} / \mathrm{s}$ is generated. A more detailed illustration of the jet including the beam characterization tools is shown in Figure 2. In order to remove particles which potentially can clog the nozzle, the sample solution is filtered by a micro-particle filter before passing through the glass nozzle. An additional tool holder is attached to the nozzle mount carrying a cerium doped YAG crystal, which is used to visualize both pump laser and $\mathrm{X}$-ray beam. In addition, a sharp silicon edge is mounted close to the nozzle to perform knife edge scans in order to determine $\mathrm{X}$-ray and pump laser beam sizes. Motorization of the stages does not only allow easy switching between the different tools but also performing different kind of scans such as knife edge scans for X-ray beam size determination or liquid jet scans to identify the optimum sample position.

\section{On-axis microscope}

The on-axis microscope is an essential part of the setup. The operation principle is based on three optical elements: a reflective microscope objective (RMO) with a drill hole of $1.5 \mathrm{~mm}$ in the center of the secondary mirror, a mirror with a drill hole deflecting the light from the RMO by $90^{\circ}$, and a tube lens (see Figure 3). The drill holes allow the X-ray beam to pass through the objective without absorption losses. The microscope image is recorded with an Allied Vision Prosilica Gigabit CCD camera with a pixel size of $4.65 \mu \mathrm{m}$, which corresponds to an effective pixel size of $\sim 0.3 \mu \mathrm{m}(\mathrm{M} \sim 15.4)$. With a numerical aperture of NA $=0.4$ of the RMO, the depth of field is about $\sim 3.4 \mu \mathrm{m}$. Therefore, the focal plane serves as an ideal positioning reference in beam direction. The position on the image itself and the non-distorted view in beam direction allows for easy positioning of all elements in horizontal and vertical direction with respect to the incident $\mathrm{X}$-ray beam.

\section{Detectors and data acquisition scheme}

X-ray absorption spectroscopy experiments can bedepending on the sample concentration-performed in either 


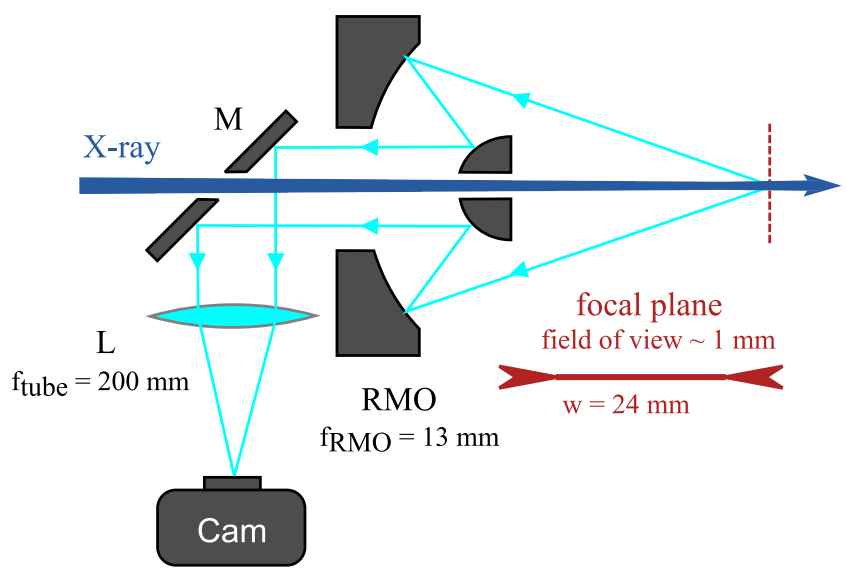

FIG. 3. Working principle of the on-axis microscope (OAM). The light from the object is collimated by the reflective microscope objective (RMO) with a focal length $f_{R M O}$ and a working distance $w$, deflected by $90^{\circ}$ by a mirror (M), passing through the tube lens (L) with a focal length of $f_{\text {tube }}$, which generates an image at the position of the CCD camera (Cam). The X-rays come from the right and pass through the drill holes in the downward deflecting mirror (M) and the secondary mirror of the RMO. The setup is adjusted such along the beam direction that the focal plane of the X-ray focusing optics matches the focal plane of the RMO.

X-ray transmission or X-ray fluorescence mode. The setup at beamline P11 features a silicon PIN diode in transmission geometry for $I_{0}$ measurements and calibration purposes. For X-ray fluorescence detection, two avalanche photodiodes (APDs) are installed to account for thin sample conditions. ${ }^{33}$ The first one measures the X-ray fluorescence from the sample solution, while the second one measures the X-ray fluorescence from a metal foil, which is placed behind the sample and used for single shot $I_{1}$ calibration. The influence of energy dependent absorption effects by the sample on $I_{1}$ compared to $I_{0}$ is typically less than $0.5 \%$ at low sample concentrations and therefore negligible. The signal definition is given in the Appendix.

In order to obtain a full XAFS spectrum, the double crystal monochromator is scanned stepwise through the desired energy range while measuring the incident number of photons and X-ray fluorescence intensity from the sample for each data point.
Alternatively, a software-synchronized continuous movement of undulator and monochromator can be used to further decrease the total measurement time.

\section{I0 measurements}

A silicon PIN diode (S9724-010 Hamamatsu) is used for $I_{0}$ determination. The diode features an active area of $10 \times 10 \mathrm{~mm}^{2}$ and is used to monitor intensity changes of the $\mathrm{X}$-ray beam on millisecond time scales and to normalize the fluorescence data with respect to the incident photon flux as a function of the X-ray energy. The diode has a thickness of $10 \mu \mathrm{m}$ and can therefore be easily used in transmission geometry for energies $>5 \mathrm{keV}$. The diode is shielded with $25 \mu \mathrm{m}$ thick black kapton foil.

The X-ray induced photocurrent in silicon can be used to determine the incident photon flux $\varphi$ according to Ref. 34.

\section{X-ray fluorescence measurements}

For the X-ray fluorescence measurements, we utilize avalanche photodiodes from FMB Oxford (APD001 model) with their corresponding pulse processing unit. ${ }^{35,36}$ The active silicon layer of one APD head has a size of $5 \times 5 \mathrm{~mm}^{2}$ with a thickness of $110 \mu \mathrm{m}$ and thus provides an efficient X-ray fluorescence detection for X-ray energies of up to $12 \mathrm{keV}$. The pulse processing unit further converts the analog voltage signal to a discriminator range of -0.2 to $5 \mathrm{~V}$ and provides digital output pulses whenever X-ray photons are detected within or above preset voltage thresholds in the discriminator. Both signals, the analog voltage signal and the digital output pulses, can be utilized for data acquisition. Utilization of the analog signal requires so-called gated integrators, ${ }^{8}$ while the digital pulses are very well suited for signal processing with constant fraction discriminators.

\section{Signal processing and temporal gating}

The data acquisition scheme in Figure 4 illustrates two main circuits: one for the signal flow for measuring the X-ray

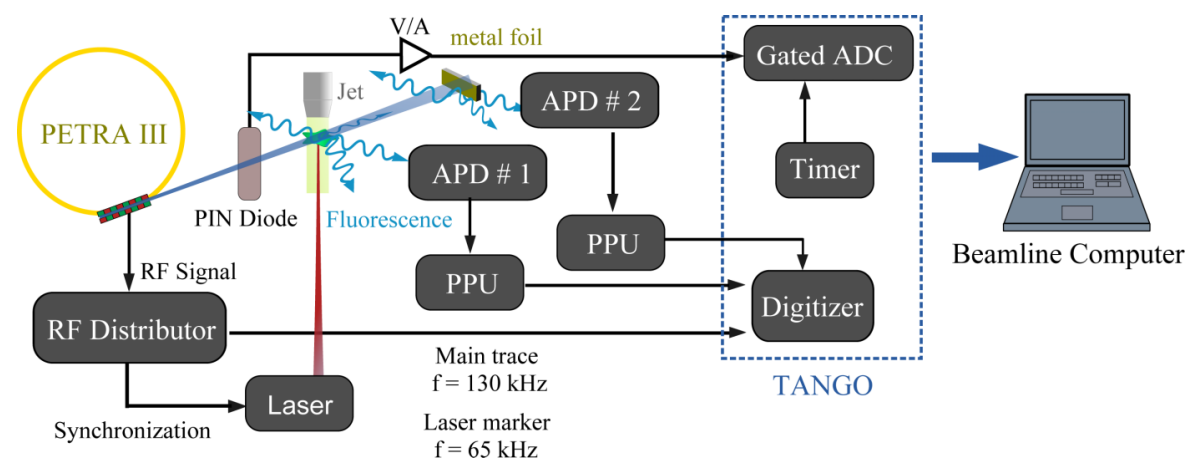

FIG. 4. Block diagram of the data acquisition circuit. RF: radio frequency; V/A: volt-amp converter/amplifier, PPU: pulse processing unit; APD: avalanche photodiode; ADC: analog-to-Digital-Converter; Timer: hardware gate generator. The left upper part illustrates the $\mathrm{I}_{0}$ signal processing for the PIN diode. The photo current is amplified and converted to a voltage signal. The voltage signal is digitized by an ADC, which is gated by a hardware timer. The lower right part shows the processing of the X-ray fluorescence signals $\mathrm{I}_{\mathrm{F}}$ and $\mathrm{I}_{1}\left(\propto \mathrm{I}_{0}\right)$. The raw voltage signals are guided into a fast digitizer, which is fed with reference signals. 
fluorescence, and the other one shows the signal flow for the $I_{0}$ and calibration measurements.

The first circuit illustrates the processing of the $\mathrm{X}$ ray fluorescence signals. The detected X-ray fluorescence photons with the APDs provide a raw voltage signal, which is proportional to the number of measured photons, e.g., if two incident X-rays simultaneously hit the active layer, the voltage signal is doubled compared to a single photon event. Following a pulse processing unit, the voltage signals are fed into a fast digitizer (model ADQ412AC, 12 bit, 2/4GS) from SP Devices for processing. The corresponding firmware and software has been developed by the European XFEL (Advanced Electronics Group, Control \& Analysis Software Group, and the FXE Group).

The digitizer is triggered by external reference signals from the PETRA III master clock. This is mandatory since the repetition rate of the laser system does not match the repetition rate of the synchrotron.

Our measurements are preferably performed in the 40 bunch mode of PETRA III (see Figure 5) where the X-ray bunches are separated by $192 \mathrm{~ns}$. Our strategy is to use always the same X-ray bunch for measurements in order to minimize noise contributions caused by X-ray beam fluctuations induced by top-up operation. Hence, the main measurement trace of the digitizer is defined by a $130 \mathrm{kHz}$ reference signal. After each trigger, the raw voltage signal of the avalanche photo diodes is summed up in a defined temporal region of interest. The latter can be comfortably set in the software from European XFEL. In order to distinguish between the pumped signal $I^{e x}(E, t)$, which denotes the measured X-ray fluorescence after laser excitation at time delay $t$, and the unpumped static signal $I^{s t}(E)$ without laser excitation, the digitizer is additionally fed with a $65 \mathrm{kHz}$ reference signal corresponding to the laser repetition rate. This signal serves as a laser marker in the software and allows separating the pumped and unpumped (static) signals. The operation of the digitizer is started by a software trigger to measure a defined number of hardware trigger pulses (main traces), which define the data acquisition time. After this number of trigger pulses is reached, the digitizer provides a mean voltage value proportional to the number of detected X-ray photons and is read out by the

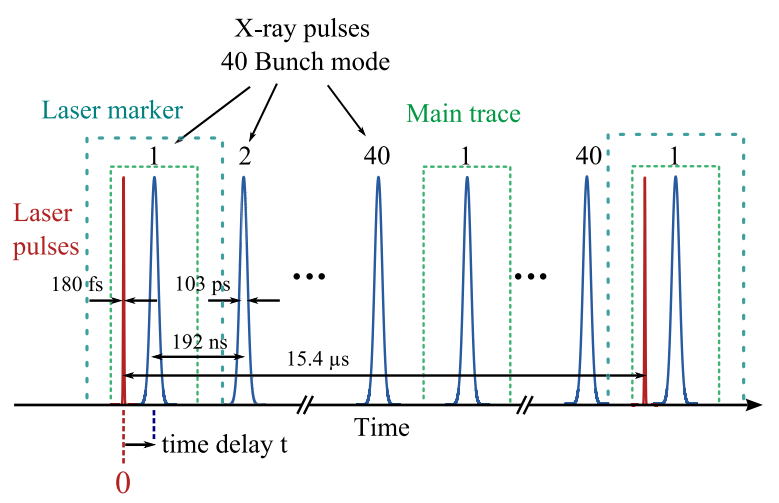

FIG. 5. Temporal structure of the data acquisition in 40 bunch mode for measuring pumped and unpumped X-ray absorption spectra simultaneously. The laser repetition rate is set to half of the revolution frequency of the synchrotron. beamline computer via TANGO, which is the standard control system utilized at the synchrotron storage ring PETRA III. More background information for the control system can be found on the TANGO Controls website. ${ }^{37}$

The second circuit in Figure 4 illustrates the processing of the measured photocurrent from the silicon PIN diode for $\mathrm{X}$-ray flux determination. The current signal is amplified and converted into a voltage signal, which is guided into a gated analog-to-digital voltage converter (ADC). The ADC averages the voltage signal over the time as utilized for the X-ray fluorescence signal gating utilizing a hardware timer. After the digitizer has acquired the preset number of triggers, the hardware timer is stopped and the averaged voltage is read out by the beamline computer.

\section{EXPERIMENTAL METHODS}

\section{A. Achievement of spatial and temporal overlap}

The feasibility to perform a rapid and reproducible spatial and temporal overlap typically decides about the success of a pump and probe experiment. In our setup, spatial overlap is achieved visually with the on-axis-microscope, while coarse temporal overlap is achieved with a fast photodiode (FPD), which is temporarily placed at the sample position.

For spatial overlap, the YAG crystal, which is located above the jet nozzle on the tool holder, is moved into the view field and the focal plane of the on-axis microscope (OAM) to visualize the X-ray beam. A digital marker in the control software is used to indicate the spot size and position of the X-ray beam in a graphical user interface. Afterwards, the scintillator is moved out and the liquid jet is moved to the marked position of the X-ray beam. The horizontal position of the liquid jet (perpendicular to the beam direction) is now scanned through the X-ray beam while the X-ray fluorescence signal from the sample is recorded. The position with the highest fluorescence signal is regarded as the optimum position and the jet is finally moved to this position.

Afterwards, the laser pump beam needs to be adjusted in vertical and horizontal direction to the center of the defined overlap position of the liquid jet and the X-ray beam. In case of visible photoluminescence of the sample solution, this can be achieved by visualizing the laser beam position in the OAM by looking at the photoluminescence.

In cases where no photoluminescence from the sample is visible, a different procedure is applied. For alignment of the vertical laser beam position, the YAG crystal is placed at the overlap position and the photoluminescence of the YAG crystal is used to visualize the laser beam position. Due to a different angle between the microscope optical axis and laser beam, which is typically ca. $48^{\circ}$, this is not possible for the horizontal alignment. Here, the correct horizontal pump laser beam position is identified by visual inspection of laser light scattered by the liquid jet.

For temporal alignment, the liquid jet is moved out of the beam and a fast photodiode (FPD), which is sensitive to both X-ray and UV/VIS radiation, is moved to the spatial overlap position. The FPD signal is monitored with a fast oscilloscope to obtain temporal overlap between laser and 
a)

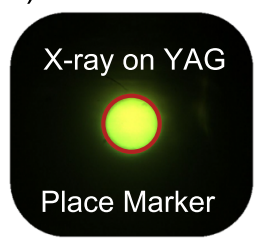

b)

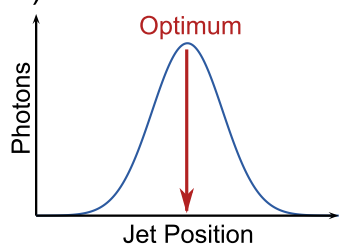

c)

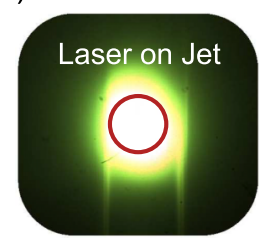

d)

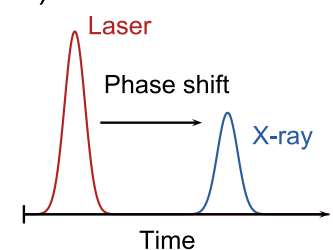

FIG. 6. (a) The X-ray beam is visualized with a scintillator in the focal plane of the on-axis microscope. A digital marker is utilized to define the size and position. (b) The liquid jet is scanned in the focal plane of the microscope to identify the optimum position by measuring the X-ray fluorescence. (c) The liquid jet is placed at the optimum horizontal position and yields a perfect agreement with the pre-defined X-ray marker. Subsequently, the laser is aligned in a way to excite the sample volume in the defined overlap region. (d) After moving the liquid jet out of the X-ray beam, a fast photodiode is moved to the spatial overlap position to obtain temporal overlap. For this purpose, the diode signal is monitored with a fast oscilloscope and the laser pulse is phase shifted temporally.

$\mathrm{X}$-ray pulses. Overlap is achieved by selecting the laser pulse, which is nearest to the $\mathrm{X}$-ray pulse by setting the correct Pockels cell trigger signal for the regenerative amplifier of the pump laser. Subsequently, the laser pulses are temporally shifted in fine steps (ps) using a phase shifter. The entire overlap strategy is summarized and illustrated in Figure 6. The temporal overlap is then fine-tuned with the pump-probe signal itself-as commonly applied in other optical pumpprobe experiments, e.g., Refs. 8 and 25. For this purpose, the temporal evolution of the pump-probe $\mathrm{X}$-ray fluorescence at a fixed X-ray energy is measured as a function of the time delay between X-ray and laser pulse.

After this temporal overlap optimization, the laser spot is scanned in both directions while recording the X-ray pumpprobe signal to fine-tune the spatial overlap. The resulting measured pump-probe fluorescence map provides a direct illustration of the spatial convolution of laser pump and X-ray probe beam (cf. Ref. 28).

\section{B. Beam feedback systems}

The storage ring PETRA III provides highly brilliant $\mathrm{X}$-ray radiation, which in principle allows for micro-beam applications due to the inherent low emittance of $1 \mathrm{~nm}$ rad only. In particular, a very large number of photons per second $\left(\leq 2 \times 10^{13} \mathrm{ph} / \mathrm{s}\right)$ can be delivered in small spot sizes of a few square micrometers to the experiment and thus offer the possibility to work with small sample amounts and small jet diameters.

In a pump and probe experiment, the spatial overlap between sample, laser spot, and X-ray spot has to be kept stable over the whole measurement time of up to several hours. Hence, to fully benefit from the outstanding microbeam properties of PETRA III, we have developed two feedback systems to guarantee high spatial stability in the order of micrometers between the jet for sample delivery, pump laser spot, and X-ray beam. Both feedback systems will be presented in the following.

\section{Laser feedback}

Laser feedback control mechanisms are typically equipped with quadrant or lateral effect sensors for beam position detection. These devices provide precise position detection with a high bandwidth. In our case, positional insta- bilities of the laser system are mainly caused by thermal drifts within the laser system itself and of the beam guiding optical elements. Such thermal drifts typically occur at subhertz frequencies and therefore do not require a fast feedback system. We have developed a feedback system operating in the few Hertz range, which is based on detecting the pump laser induced luminescence of the sample in the visible regime with our on-axis microscope. The center of gravity of the luminescence is constantly steered to the pre-defined position to maintain optimal spatial overlap. In case of no luminescence from the sample, the scattered laser light from the jet is still sufficient for adequate beam position detection.

For laser beam position control a single mirror system, whose reflecting angle in vertical and horizontal position can be adjusted with piezo ceramics with a full stroke of $23 \mu \mathrm{m}$, is installed in the laser beam path. Feasible compensated deviations are-depending on the optics and beam alignment - in the range of $100 \mu \mathrm{m}$ at the sample position.

The working principle of the optical feedback mechanism is illustrated in Figure 7 utilizing the transition metal complex $\operatorname{Ir}(\mathrm{ppy})_{3}$ as sample. We slightly misaligned the pump laser beam at the start, which is noticeable by the rather weak luminescence. The UV/VIS beam is subsequently shifted back to the preset position in the center of the liquid jet by our feedback system.

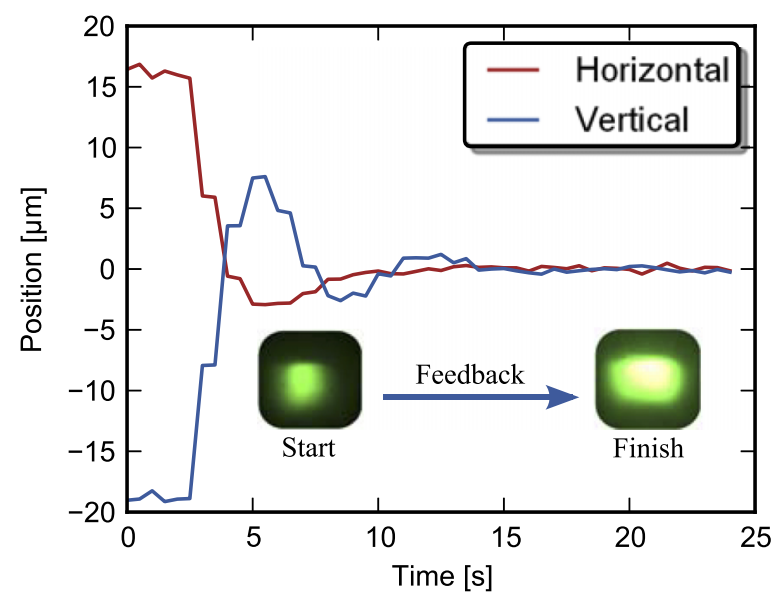

FIG. 7. Illustrated working principle of the laser feedback, where the laser beam excites the sample molecules inside the liquid jet (insets). The visible luminescence is utilized to lock the beam position in the on-axis microscope. 
In order to provide a stable and safe long-term operation, the optical feedback is automatically paused when the laser beam is blocked, e.g., with Pockels cells, and afterwards resumed when the laser exposure on the sample is active again.

This optical feedback system can be further utilized to initially align the UV/VIS beam from the outside of the experimental hutch and to optimize the spatial overlap between $\mathrm{X}$-ray and laser beam.

\section{X-ray feedback system}

The X-ray feedback control system is based on a $10 \mu \mathrm{m}$ thick Chemical Vapor Deposition (CVD) diamond foil with four quadrant electrodes on one side and a single electrode on the other side working in transmission geometry. ${ }^{38}$ Such a detector is commonly described as X-ray beam position monitor (XBPM). The electrodes consist of a $10 \mathrm{~nm}$ titan adhesive layer followed by $200 \mathrm{~nm}$ aluminum.

Compared to other materials, a diamond foil is highly transparent for X-rays and only a small fraction of the X-rays is absorbed. The absorbed photons are mainly converted into photo electrons, which induce a photo current between the front side quadrants and the backside electrodes. This current from the four individual quadrants is proportional to the absorbed X-ray photons. Hence, the ratio of the photo current between the different quadrants can be utilized to determine the center of the beam position. The working principle of the XBPM is illustrated in Figure 8. Each quadrant current $I_{A, B, C, D}$ is amplified and converted into a voltage signal by a low-noise current amplifier and afterwards digitized by a high-bandwidth analog-to-digital converter (ADC) (Model ICS-110BL from GE Intelligent Platforms) to measure the currents in defined intervals. The normalized beam position can be calculated according to

$$
\begin{aligned}
& X=\frac{\left(I_{B}+I_{D}\right)-\left(I_{A}+I_{C}\right)}{\sum I}, \\
& Y=\frac{\left(I_{A}+I_{B}\right)-\left(I_{C}+I_{D}\right)}{\sum I} .
\end{aligned}
$$

At beamline P11, the XBPM is placed at the position of the secondary source in a vacuum chamber located on the same granite block as the experiment. Vacuum conditions are
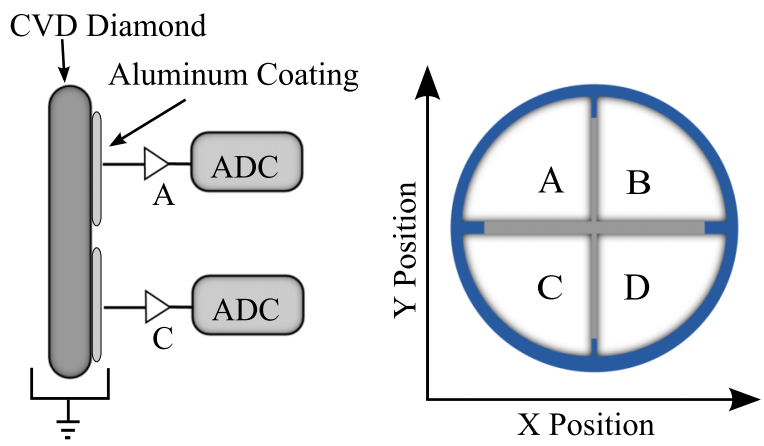

FIG. 8. Working principle (side view) and geometry (front view) of a quadrant detector with denoted areas. ${ }^{49}$ ADC: analog-to-digital converter; A,B,C,D: quadrant notation. required for the XBPM in order to prevent the electrodes from $\mathrm{X}$-ray mediated oxidation. The resolution of the XBPM has been characterized to be better than $2 \mu \mathrm{m}$. Due to the very high thermal stability conditions in the P11 experimental hutch, no deviations between the beam positions as detected with the XBPM and behind the second refocusing KB-system with the on-axis microscope looking at the YAG fluorescence screen located at the sample position could be observed. X-ray beam position corrections in horizontal direction are performed by a piezo-motor driven rotation stage of the first KB-mirror system located in the optics hutch, while the pitch angle of the second crystal in the monochromator is utilized to control the vertical position.

Exemplarily, we have measured the X-ray beam position of both horizontal and vertical direction with a bandwidth of $2 \mathrm{~Hz}$ in order to illustrate the thermal drift of the beam position (see Figure 9) and to demonstrate the utilizability of the feedback control system for XAFS experiments. In the measurements shown in Figure 9, a deadband threshold of $\sim 3.5 \mu \mathrm{m}$ was applied in the control algorithm, meaning that no control action is taken while the measured beam position, either horizontal or vertical, is within the deadband region. We have to emphasize that the beam deviations in vertical direction are further reduced by a factor of 10 due to the refocusing KB-system shortly before the XAFS setup.

In conclusion, the X-ray feedback system allows performing X-ray absorption spectroscopy measurements with micron-sized beams and guarantees excellent spatial overlap between X-ray and laser beam. Without a closed-loop control of the X-ray beam position and the laser beam position such experiments would not be possible.

Without feedback, we observe large beam position changes at the position of the XBPM, which can be as large as several hundreds of microns, in particular, during energy scans.
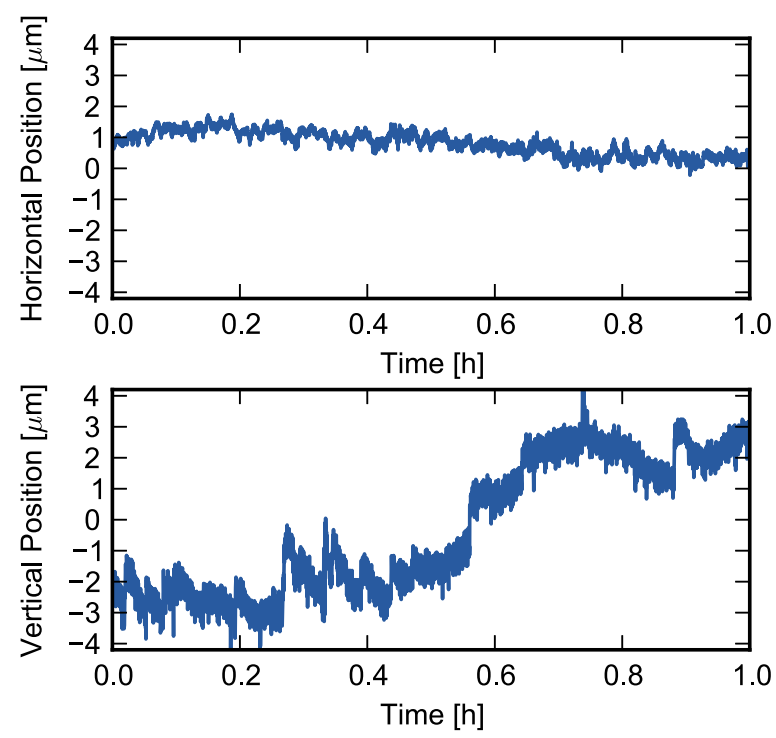

FIG. 9. Measured thermal drift of the X-ray beam position in the horizontal (top) and vertical direction (bottom) with the X-ray beam feedback on. 


\section{TIME-RESOLVED PUMP-PROBE XAFS OF A CHARGE TRANSFER REACTION}

\section{A. Photoinduced metal-to-ligand charge transfer in fac-Ir(ppy $)_{3}$}

The transition metal complex fac-Ir(ppy) 3 (ppy = 2-phenyl pyridine anion) is a prominent phosphorescence emitter suitable for OLED applications and has therefore been extensively studied over the last decade (see Figure 10 (a)). ${ }^{39-44}$ The emitting triplet state has been reported as a metal-to-ligandcharge transfer state. Since time-resolved XAFS is sensitive to electronic changes and thus provides significant changes in absorption in the energy region near the absorption edge, we have chosen fac- $\operatorname{Ir}(\mathrm{ppy})_{3}$ as our first molecular system to demonstrate the performance of our setup by visualizing the metal-to-ligand-charge transfer.

The excited state dynamics of fac-Ir(ppy $)_{3}$ according to Ref. 43 are briefly illustrated in Figure 10(b) and described in the following. Upon photoexcitation of the singlet metal-toligand charge transfer band $\left({ }^{1} \mathrm{MLCT}\right)$, electron density from the metal valence orbitals is transferred to the $\pi^{*}$ orbitals on the surrounding ppy ligands (Figure 10(c)). The metal valence orbital occupancy changes from $5 \mathrm{~d}^{6}$ to $5 \mathrm{~d}^{5}$. The excited system further undergoes intersystem crossing (ISC) on a femtosecond time scale to the triplet state $\left({ }^{3} \mathrm{MLCT}\right)$. The triplet state is fairly long-living and decays on a microsecond time scale to the ground state via visible luminescence. ${ }^{43}$

For our measurements, the fac-Ir(ppy) ${ }_{3}$ complex was dissolved in dimethyl sulfoxide at a concentration of $2 \mathrm{mM}$. $\mathrm{X}$-ray spectroscopic measurements were performed with an energy resolution of $\Delta \mathrm{E} / \mathrm{E}=1.3 \times 10^{-4}$ at $12 \mathrm{keV}$ (Si-111). The X-ray beam was focused down to the minimum achievable spot size $\left(4 \times 9 \mu \mathrm{m}^{2}\right)$ by the second KB-system. The jet diameter was chosen to be $200 \mu \mathrm{m}$ and the liquid jet velocity was around $7 \mathrm{~m} / \mathrm{s}$.

All absorption spectra were measured at room-temperature accumulating the X-ray fluorescence yield for $\sim 8.2 \times 10^{12}$ incident $\mathrm{X}$-ray photons onto the sample per data point. The energy region before and at the absorption edge was probed with fine step sizes of $0.5 \mathrm{eV}$. Above the absorption edge,

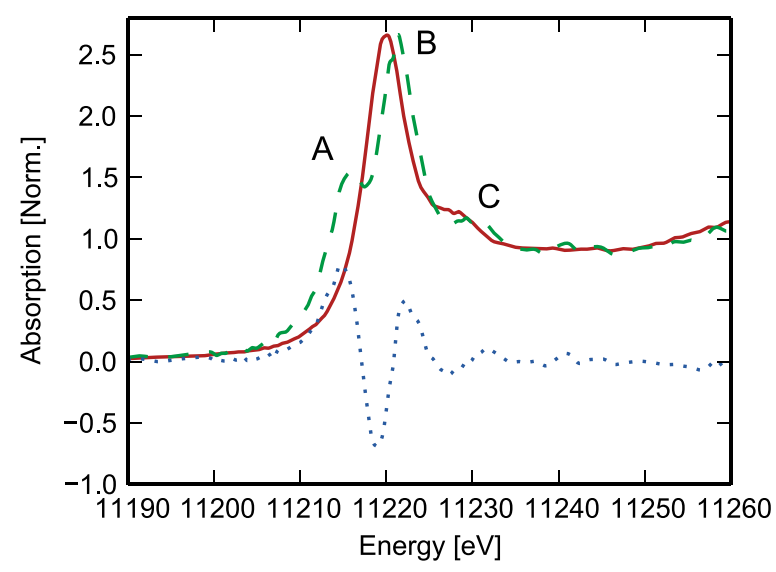

FIG. 11. Measured static absorption spectrum of $\operatorname{Ir}(\text { ppy })_{3}$ dissolved in dimethyl sulfoxide (solid curve), normalized transient difference spectrum (dotted curve), and reconstructed excited state spectrum (dashed curve) at the $\mathrm{L}_{3}$ edge of iridium. The transient difference spectrum was measured at a time delay of $\sim 200 \mathrm{ps}$.

the step size was gradually increased to $4 \mathrm{eV}$ in order to cover a larger energy range for edge-jump normalization. For the simultaneously recorded pump-probe spectrum, the sample was photoexcited with $\sim 180$ fs short laser pulses at a wavelength of $343 \mathrm{~nm}$. The laser beam was focused down to a spot size approximately matching the liquid jet size and we applied a fluence of $\leq 12 \mathrm{~mJ} / \mathrm{cm}^{2}$ onto the sample.

At the X-ray energy of $\sim 11215 \mathrm{eV}$, a so-called time scan was performed by changing the delay time between pump and probe pulse in picosecond steps. All other experimental parameters were kept the same.

\section{B. Results}

Our measured pump and probe absorption spectra at the $\mathrm{L}_{3}$ edge of iridium are shown in Figure 11. The solid (red) curve represents the static absorption spectrum, while the dotted (blue) curve is the transient difference spectrum measured at a time delay of $\sim 200$ ps after photoexcitation and a)

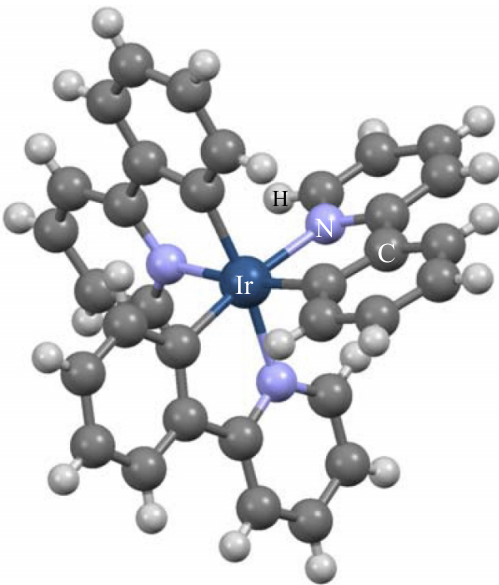

b)

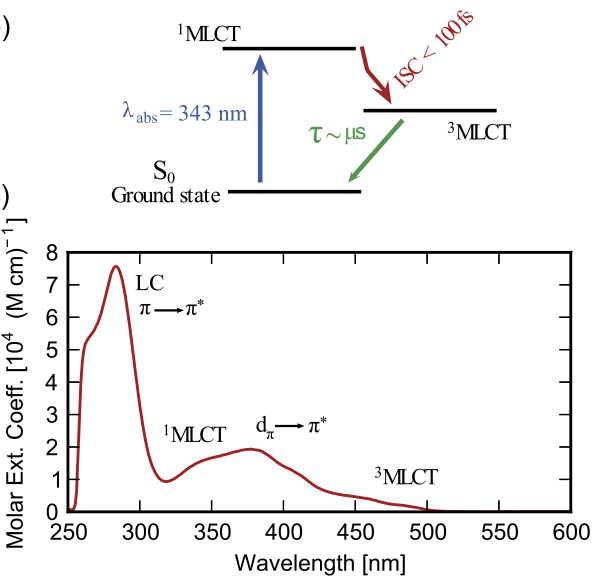

FIG. 10. (a) Structure of the green phosphorescence emitter complex fac-Ir(ppy) 3 ; Ir: iridium; N: nitrogen; C: carbon; H: hydrogen (reproduced from Ref. 39 with mercury); (b) energy diagram and excited state dynamics ${ }^{43}$ of the charge transfer reaction in Ir(ppy) 3 ; (c) measured steady-state UV/VIS absorption spectrum of the ground state. LC: ligand-centered transition; MLCT: metal-to-ligand charge transfer. Assignments according to Refs. 43 and 44. 
scaled by a factor of $1 / 0.165$ (see below) to account for the photoexcited fraction of the sample molecules. The excited state absorption spectrum-represented by a dashed (green) curve-was then derived by adding the transient difference spectrum accounting for a full photoexcitation yield to the ground state spectrum.

It is clearly visible that the most pronounced photoinduced changes in absorption are in the vicinity of the edge resonance. For a further discussion, we have denoted the pronounced spectral resonances as A, B, and C.

We attribute the arising spectral resonance $\mathrm{A}$, which is linked to the first maximum in the transient difference spectrum, to the absorption of excited photoelectrons in the photoinduced vacancy of the metal valence orbitals. ${ }^{16,45}$ In turn, the spectral resonance B in both static and excited state spectrum can be assigned to the absorption of excited core electrons in the remaining empty metal d-orbitals of the $5 \mathrm{~d}^{6}$ ground state configuration. It is noticeable that this resonance is blue-shifted in energy after photoexcitation, which together with the appearance of feature A, provides evidence of a successfully photoinduced metal-to-ligand charge transfer. In addition, we can attribute the $\mathrm{C}$ features with regard to other $\mathrm{X}$-ray absorption spectroscopy experiments on ruthenium complexes as multiple-scattering resonances. ${ }^{16,46}$

We derived a photoexcitation yield of $\sim 16.5 \%$ from our iridium experiments by scaling our transient difference spectrum according to our experimental results of $\operatorname{Ir}(\mathrm{ppy})_{3}$ provided in Ref. 23 where an ionic approximation of the metal valence orbitals is used. Considering that the measured ground state spectrum reflects a $5 \mathrm{~d}^{6}$ configuration and the excited triplet state is linked to a $5 \mathrm{~d}^{5}$ configuration, the transient difference spectrum is scaled in a way that the integrated absorption of feature A and B in the excited state spectrum corresponds to $5 / 4$ of their counterpart in the ground state spectrum. ${ }^{47}$

Knowledge of the spin-orbit coupling constant of iridium and the energy difference between features B and A in the excited state spectrum provides information on the ligand field splitting 10Dq. ${ }^{45}$ Thus, if we consider a spin-orbit coupling interaction of ca. $0.55 \mathrm{eV}$ (see, for example, Nozaki ${ }^{42}$ ) and an extracted $\Delta \mathrm{E}$ of $\sim 5.9 \pm 0.2 \mathrm{eV}$, we can determine the $10 \mathrm{Dq}$ parameter in the triplet state as $\sim 6.45 \pm 0.20 \mathrm{eV}$.

In addition, we could derive information on the X-ray bunch length of PETRA III from our $\operatorname{Ir}(\mathrm{ppy})_{3}$ measurements since the formation of the long-living triplet state occurs on a femtosecond time scale.

For this purpose, we followed the temporal convolution of X-ray and laser beam by measuring the transient signal near feature A (see Figure 11) since we observed the most pronounced photoinduced changes around this X-ray energy. The outcome is shown in Figure 12. If we consider Gaussian shaped pulses and a Gaussian shaped synchronization contribution, the convolution of these profiles will also result in a Gaussian profile and we can derive the temporal resolution of the experiment by

$$
\sigma_{\text {Total }}=\sqrt{\sigma_{X-\text { ray }}^{2}+\sigma_{\text {Laser }}^{2}+\sigma_{\text {Sync }}^{2}} .
$$

We fitted the experimental data with a cumulative distribution function as step function and obtained a temporal resolution of

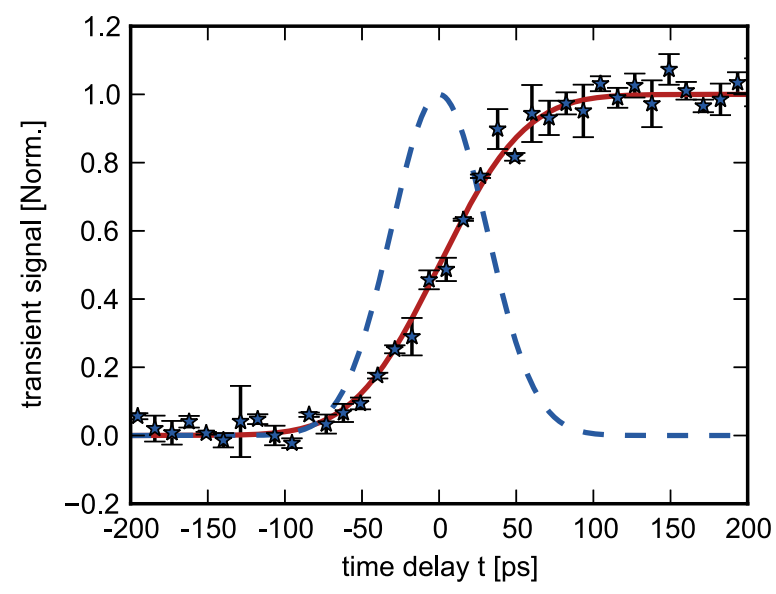

FIG. 12. Measured temporal evolution of the pump-probe absorption signal of $\operatorname{Ir}(\mathrm{ppy})_{3}$ dissolved in dimethyl sulfoxide at a fixed X-ray energy. A cumulative distribution function for the convolution of X-ray and laser pulse (solid curve) was fitted to the data and the derivative (dashed curve) represents the approximated temporal shape of the X-ray pulse (Gaussian). We characterized the temporal resolution of the experiment to $\sim 103 \pm 2 \mathrm{ps}$ (FWHM).

$\approx 103 \pm 2$ ps (FWHM). Since the synchronization contribution $(\leq 1 \mathrm{ps} \mathrm{rms}$ ) and laser pulse duration are negligible, this magnitude represents the X-ray bunch length of PETRA III. The Gaussian shaped synchrotron pulse can be obtained as the derivative of the step function and is shown in Figure 12 as dashed (blue) curve.

\section{CONCLUSION AND OUTLOOK}

We have developed, implemented, and successfully tested a novel setup for time-resolved X-ray absorption experiments at beamline P11 at the PETRA III synchrotron storage ring in Hamburg.

The usage of micrometer sized beams as well as the high photon flux available at PETRA III allows for a significant reduction of the required sample amount, which makes the setup also very well-suited for experiments with valuable samples such as metalloproteins, where only small sample amounts are available. To provide stable overlap between the X-ray beam, the laser pump beam, and the jet for sample delivery, special care was taken for the design of the experiment. The refocusing $\mathrm{KB}$ system and the interaction point are located on a 10 ton granite block and we have developed and implemented beam position control systems for the X-ray and laser beam, which can operate 24/7 and thus ensure spatial overlap over the whole measurement time.

In addition, the high flux density available at the experiment allows recording of a full time-resolved XAFS spectrum covering an energy range of several hundreds of $\mathrm{eV}$ in a reasonable time ( $\sim 90 \mathrm{~min})$.

We have successfully demonstrated the feasibility of our setup with benchmark experiments on the green phosphorescence emitter complex fac-Ir(ppy $)_{3}$. The results present a direct proof of the photoinduced charge transfer reaction and the ligand field splitting parameter of the triplet state was determined experimentally as $\sim 6.45 \pm 0.20 \mathrm{eV}$. 
In addition, we followed the temporal evolution of the transient signal at a fixed X-ray energy, which is linked to the photoinduced charge transfer, to determine the X-ray bunch length of PETRA III as $\sim 103 \pm 2$ ps.

Just recently, the setup has also been used for timeresolved pump and probe liquid diffraction measurements. For this purpose, the setup was equipped with a beam stop directly after the liquid jet system. Removing the metal foil for $I_{0}$ calibration allows for the usage of the $6 \mathrm{M}$ Pilatus detector directly behind the XAFS setup for diffraction measurements.

\section{ACKNOWLEDGMENTS}

The authors thank the staff of beamline P11 at PETRA III. Part of this work was supported by the Virtual Institute VH-VI-403 of the Helmholtz Association. Portions of this research were funded by the Hamburg Centre for Ultrafast Imaging (CUI), the DFG via Grant No. SFB925/TPA4, and by European XFEL. A. Meents and C. Bressler acknowledge funding by the EU-funded Cluster of Research Infrastructures EUCALL within PUCCA and HIREP.

\section{APPENDIX: SIGNAL DEFINITION}

The following definitions are introduced according to Refs. 6 and 48. The static absorption signal $A^{s t}(E)$ and the time-resolved pumped signal $A^{e x}(E, t)$ are derived via the measured fluorescence intensity $I^{s t}(E)$ without laser excitation and the fluorescence signal $I^{e x}(E, t)$ prior to laser excitation as follows:

$$
\begin{aligned}
A^{s t}(E) & =\frac{I^{s t}(E)}{I_{0}(E)}, \\
A^{e x}(E, t) & =\frac{I^{e x}(E, t)}{I_{0}(E)},
\end{aligned}
$$

where $\mathrm{I}_{0}(\mathrm{E})$ is the incident intensity and $t$ is the time delay between $\mathrm{X}$-ray and laser pulse. The transient difference signal $\Delta T(E, t)$ can be introduced as

$$
\Delta T(E, t)=\frac{I^{e x}(E, t)-I^{s t}(E)}{I_{0}(E)} .
$$

${ }^{1}$ C. Bressler and M. Chergui, Annu. Rev. Phys. Chem. 61, 263 (2010).

${ }^{2}$ L. X. Chen, Angew. Chem., Int. Ed. 43, 2886 (2004).

${ }^{3}$ L. X. Chen, Annu. Rev. Phys. Chem. 56, 221 (2005).

${ }^{4}$ C. J. Milne, V.-T. Pham, W. Gawelda, R. M. Van Der Veen, A. El Nahhas, S. L. Johnson, P. Beaud, G. Ingold, F. Lima, D. A. Vithanage, M. Benfatto, D. Grolimund, C. Borca, M. Kaiser, A. Hauser, R. Abela, C. Bressler, and M. Chergui, J. Phys.: Conf. Ser. 190, 012052 (2009).

${ }^{5}$ M. Saes, C. Bressler, R. Abela, D. Grolimund, S. L. Johnson, P. A. Heimann, and M. Chergui, Phys. Rev. Lett. 90, 047403 (2003).

${ }^{6}$ C. Bressler, M. Saes, M. Chergui, D. Grolimund, R. Abela, and P. Pattison, J. Chem. Phys. 116, 2955 (2002).

${ }^{7}$ N. Huse, M. Khalil, T. K. Kim, A. L. Smeigh, L. Jamula, J. K. Mccusker, and R. W. Schoenlein, J. Phys.: Conf. Ser. 148, 012043 (2009).

${ }^{8}$ M. Saes, F. Van Mourik, W. Gawelda, M. Kaiser, M. Chergui, C. Bressler, D. Grolimund, R. Abela, T. E. Glover, P. A. Heimann, R. W. Schoenlein, S. L. Johnson, A. M. Lindenberg, and R. W. Falcone, Rev. Sci. Instrum. 75, 24 (2004).

${ }^{9}$ G. Smolentsev, A. Guda, X. Zhang, K. Haldrup, E. Andreiadis, M. ChavarotKerlidou, S. E. Canton, M. Nachtegaal, V. Artero, and V. Sundstrom, J. Phys. Chem. C. Nanomater. Interfaces 117, 17367 (2013).
${ }^{10}$ W. Gawelda, C. Bressler, M. Saes, M. Kaiser, A. N. Tarnovsky, D. Grolimund, S. L. Johnson, R. Abela, and M. Chergui, Phys. Scr. T115, 102 (2005).

${ }^{11}$ M. W. Mara, N. E. Jackson, J. Huang, A. B. Stickrath, X. Zhang, N. A. Gothard, M. A. Ratner, and L. X. Chen, J. Phys. Chem. B 117, 1921 (2013).

${ }^{12}$ M. Khalil, M. A. Marcus, A. L. Smeigh, K. Mccusker, H. H. W. Chong, R. W. Schoenlein, and J. K. Mccusker, J. Phys. Chem. A 110, 38 (2006).

${ }^{13}$ D. J. Thiel, P. Livins, E. A. Stern, and A. Lewis, Nature 362, 40 (1993).

${ }^{14}$ W. Gawelda, V. T. Pham, M. Benfatto, Y. Zaushitsyn, M. Kaiser, D. Grolimund, S. L. Johnson, R. Abela, A. Hauser, C. Bressler, and M. Chergui, Phys. Rev. Lett. 98, 057401 (2007).

${ }^{15}$ L. X. Chen, G. B. Shaw, I. Novozhilova, T. Liu, G. Jennings, K. Attenkofer, G. J. Meyer, and P. Coppens, J. Am. Chem. Soc. 125, 7022 (2003).

${ }^{16}$ W. Gawelda, M. Johnson, F. M. F. de Groot, R. Abela, C. Bressler, and M. Chergui, J. Am. Chem. Soc. 128, 5001 (2006).

${ }^{17}$ R. Ortega, A. Carmona, I. Llorens, and P. L. Solari, J. Anal. At. Spectrom. 27, 2054 (2012).

${ }^{18}$ G. Smolentsev and V. Sundström, Coord. Chem. Rev. 304-305, 117 (2015).

${ }^{19}$ S. Bordiga, E. Groppo, G. Agostini, J. van Bokhoven, and C. Lamerti, Chem. Rev. 113, 1736 (2013).

${ }^{20}$ J. J. Rehr, Rev. Mod. Phys. 72, 621 (2000).

${ }^{21}$ E. A. Stern, Phys. Rev. B 11, 2795 (1975).

${ }^{22}$ D. C. Koningsberger and R. Prins, X-Ray Absorption: Principles, Applications, Techniques of EXAFS, SEXAFS and XANES (John Wiley \& Sons, 1988).

${ }^{23}$ D. Göries, Ph.D. thesis, University of Hamburg, 2014.

${ }^{24}$ B. Dicke, Ph.D. thesis, University of Hamburg, 2013.

${ }^{25}$ F. A. Lima, C. J. Milne, D. C. V. Amarasinghe, M. H. Rittmann-Frank, R. M. Van Der Veen, M. Reinhard, V. T. Pham, S. Karlsson, S. L. Johnson, D. Grolimund, C. Borca, T. Huthwelker, M. Janousch, F. Van Mourik, R. Abela, and M. Chergui, Rev. Sci. Instrum. 82, 063111 (2011).

${ }^{26}$ G. Smolentsev, A. Guda, M. Janousch, C. Frieh, G. Jud, F. Zamponi, M. Chavarot-Kerlidou, V. Artero, J. van Bockhoven, and M. Nachtegaal, Faraday Discuss. 171, 259 (2014).

${ }^{27}$ A. M. March, A. Stickrath, G. Doumy, E. P. Kanter, B. Krässig, S. H. Southworth, K. Attenkofer, C. a Kurtz, L. X. Chen, and L. Young, Rev. Sci. Instrum. 82, 073110 (2011).

${ }^{28}$ T. Sato, S. Nozawa, K. Ichiyanagi, A. Tomita, M. Chollet, H. Ichikawa, H. Fujii, S. I. Adachi, and S. Y. Koshihara, J. Synchrotron Radiat. 16, 110 (2008).

${ }^{29}$ C. Ulbricht, B. Beyer, C. Friebe, A. Winter, and U. S. Schubert, Adv. Mater. 21, 4418 (2009)

${ }^{30} \mathrm{H}$. Yersin, Highly Efficient OLEDs with Phosphorescent Materials, 1st ed. (Wiley-VCH Verlag GmbH \& Co. KGaA, 2008).

${ }^{31}$ K. Balewski, W. Brefeld, W. Decking, H. Franz, and E. Weckert, PETRA III: A Low Emittance Synchrotron Radiation Source Technical Design Report, (Hamburg, 2004).

${ }^{32}$ M. Schlie, Ph.D. thesis, University of Hamburg, 2013.

${ }^{33}$ J. Jaklevic, J. A. Kirby, M. P. Klein, A. S. Robertson, G. S. Brown, and P. Eisenberger, Solid State Commun. 88, 1105 (1993).

${ }^{34}$ R. L. Owen, J. M. Holton, C. Schulze-Briese, and E. F. Garman, J. Synchrotron Radiat. 16, 143 (2009).

${ }^{35}$ FMB Oxford, Issue 3.0 DOCM0101, 2011.

${ }^{36}$ FMB Oxford, Issue 2.0 DOCM0102, 2010.

${ }^{37}$ TANGO Community, http://www.tango-controls.org/, 2015.

${ }^{38} \mathrm{P}$. Leban, D. Tinta, and C. Pradervand, in Proceeding of IPAC'10, Kyoto, Japan, 2010, http://www.jacow.org, pp. 1077-1079.

${ }^{39}$ J. Breu, P. Stössel, S. Schrader, A. Starukhin, W. J. Finkenzeller, and H. Yersin, Chem. Mater. 17, 1745 (2005).

${ }^{40}$ J. Fine, K. Diri, A. I. Krylov, C. Nemirow, Z. Lu, and C. Wittig, Mol. Phys. 110, 37 (2012).

${ }^{41}$ W. J. Finkenzeller and H. Yersin, Chem. Phys. Lett. 377, 299 (2003).

${ }^{42}$ K. Nozaki, J. Chin. Chem. Soc. 53, 101 (2006).

${ }^{43}$ G. J. Hedley, A. Ruseckas, and I. D. W. Samuel, Chem. Phys. Lett. 450, 292 (2008).

${ }^{44}$ T. Hofbeck and H. Yersin, Inorg. Chem. 49, 9290 (2010).

${ }^{45}$ T. K. Sham, J. Am. Chem. Soc. 105, 2269 (1983).

${ }^{46}$ I. Alperovich, G. Smolentsev, D. Moonshiram, J. W. Jurss, J. J. Concepcion, T. J. Meyer, A. Soldatov, and Y. Pushkar, J. Am. Chem. Soc. 133, 15786 (2011).

${ }^{47}$ M. Saes, Ph.D. thesis, École polytechnique fédérale de Lausanne, 2004.

${ }^{48}$ W. Gawelda, Ph.D. thesis, École polytechnique fédérale de Lausanne, 2006.

${ }^{49}$ B. Steffen, private communication (2012). 